\title{
Quantitative versus qualitative blood amount assessment as a predictor for shunt-dependent hydrocephalus following aneurysmal subarachnoid hemorrhage
}

\author{
*Sergio García, MD, ${ }^{1}$ Ramon Torné, MD, PhD, ${ }^{1}$ Jhon Alexander Hoyos, MD, ${ }^{1}$ \\ Ana Rodríguez-Hernández, MD, PhD, ${ }^{2}$ Sergio Amaro, MD, PhD, ${ }^{3}$ Laura Llull, MD, PhD, ${ }^{3}$ \\ Antonio López-Rueda, MD, ${ }^{4}$ and Joaquim Enseñat, MD, PhD ${ }^{1}$ \\ 'Department of Neurological Surgery, Hospital Clinic de Barcelona, University of Barcelona; ${ }^{2}$ Department of Neurological \\ Surgery, Germans Trias i Pujol University Hospital; ${ }^{3}$ Comprehensive Stroke Center, Department of Neurology, Hospital Clinic \\ de Barcelona, University of Barcelona and August Pi I Sunyer Biomedical Research Institute (IDIBAPS); and ${ }^{4}$ Department of \\ Radiology, Hospital Clinic de Barcelona, University of Barcelona, Barcelona, Spain
}

\begin{abstract}
OBJECTIVE Reliable tools are lacking to predict shunt-dependent hydrocephalus (SDHC) development after aneurysmal subarachnoid hemorrhage (aSAH). Quantitative volumetric measurement of hemorrhagic blood is a good predictor of SDHC but might be impractical in the clinical setting. Qualitative assessment performed using scales such as the modified Fisher scale (mFisher) and the original Graeb scale (oGraeb) is easier to conduct but provides limited predictive power. In between, the modified Graeb scale (mGraeb) keeps the simplicity of the qualitative scales yet adds assessment of acute hydrocephalus, which might improve SDHC-predicting capabilities. In this study the authors investigated the likely capabilities of the $\mathrm{mGraeb}$ and compared them with previously validated methods. This research also aimed to define a tailored $\mathrm{mGraeb}$ cutoff point for SDHC prediction.
\end{abstract}

METHODS The authors performed retrospective analysis of patients admitted to their institution with the diagnosis of aSAH between May 2013 and April 2016. Out of 168 patients, 78 were included for analysis after the application of predefined exclusion criteria. Univariate and multivariate analyses were conducted to evaluate the use of all 4 methods (quantitative volumetric assessment and the mFisher, oGraeb, and mGraeb scales) to predict the likelihood of SDHC development based on clinical data and blood amount assessment on initial CT scans.

RESULTS The mGraeb scale was demonstrated to be the most robust predictor of SDHC, with an area under the curve (AUC) of 0.848 (95\% $\mathrm{Cl} 0.763-0.933)$. According to the AUC results, the performance of the mGraeb scale was significantly better than that of the oGraeb scale $\left(\chi^{2}=4.49 ; p=0.034\right)$ and mFisher scale $\left(\chi^{2}=7.21 ; p=0.007\right)$. No statistical difference was found between the AUCs of the mGraeb and the quantitative volumetric measurement models $\left(\chi^{2}\right.$ $=12.76 ; p=0.23)$, but $m$ Graeb proved to be the simplest model since it showed the lowest Akaike information criterion (66.4), the lowest Bayesian information criterion (71.2), and the highest $\mathrm{R}^{2}$ Nagelkerke coefficient $(39.7 \%)$. The initial $m G$ raeb showed more than $85 \%$ specificity for predicting the development of SDHC in patients presenting with a score of 12 or more points.

CONCLUSIONS According to the authors' data, the mGraeb scale is the simplest model that correlates well with SDHC development. Due to limited scientific evidence of treatments aimed at SDHC prevention, we propose an mGraeb score higher than 12 to identify patients at risk with high specificity. This mGraeb cutoff point might also serve as a useful prognostic tool since patients with SDHC after aSAH have worse functional outcomes.

https://thejns.org/doi/abs/10.3171/2018.7.JNS18816

KEYWORDS aneurysmal subarachnoid hemorrhage; hydrocephalus; shunt; Graeb scale; volumetry; vascular disorders

ABBREVIATIONS AIC = Akaike information criterion; $\mathrm{aSAH}=$ aneurysmal $\mathrm{SAH} ; \mathrm{AUC}=$ area under the curve $; \mathrm{BIC}=$ Bayesian information criterion; $\mathrm{CV}=$ cisternal volume; $\mathrm{EVD}=$ external ventricular drain; IV = intraventricular volume; IVH = intraventricular hemorrhage; $\mathrm{mFisher} \mathrm{=} \mathrm{modified} \mathrm{Fisher} \mathrm{scale;} \mathrm{mGraeb}=$ modified Graeb scale; $\mathrm{mRS}=$ modified Rankin Scale; oGraeb = original Graeb scale; ROC = receiver operating characteristic; $\mathrm{ROI}=$ region of interest; $\mathrm{SAH}=$ subarachnoid hemorrhage; $\mathrm{SDHC}=$ shuntdependent hydrocephalus; TV = total volume; WFNS = World Federation of Neurosurgical Societies.

SUBMITTED March 28, 2018. ACCEPTED July 19, 2018.

INCLUDE WHEN CITING Published online December 21, 2018; DOI: 10.3171/2018.7.JNS18816.

${ }^{*}$ S.G. and R.T. contributed equally to this work. 
$\mathrm{H}$ YDROCEPHALUS is a common complication after subarachnoid hemorrhage (SAH). Although rates vary widely among classic reports, acute hydrocephalus is currently estimated to occur in around 30\% of SAH cases, ${ }^{4,12,15}$ while chronic shunt-dependent hydrocephalus (SDHC) ends up affecting 20\% of patients. ${ }^{30,40}$ Patients suffering from SDHC have been identified as a cognitively and functionally impaired cohort within the group of SAH survivors. ${ }^{29,36}$ Therefore, tools for early recognition of patients at risk for this type of secondary hydrocephalus could translate into proper treatment and improved patient outcomes. However, there are few treatments that may prevent SDHC after SAH, and those available do not have solid scientific evidence to back them up. Ideally, if a given treatment is not strongly recommended, such a treatment should not be applied unless there is some certainty that the patient will develop SDHC. Therefore, there is a need for tools for predicting SDHC after SAH with as much specificity as possible.

Many efforts have been made to find solid predictors of SDHC following SAH. Several factors, such as older age, female sex, acute hydrocephalus, intraventricular hemorrhage (IVH), focal ischemic deficits, symptomatic vasospasm, mechanical ventilation on admission, posterior circulation aneurysm, and endovascular treatment, have been linked to higher incidence of SDHC. . $^{8,12,30}$ Among all of these predictive factors, IVH has been identified as an independent predictor of SDHC, mortality, and functional outcome and has been thoroughly investigated as it is suspected to lie behind both occlusive and nonocclusive mechanisms leading to hydrocephalus. ${ }^{24,34}$ Actually, measuring the real amount of intraventricular blood (volumetry) in early CT exams has proven to be an accurate predictor of SDHC. $18,20,23$

Several radiological scales for the measurement of the amount of blood visible on CT have been implemented in order to predict functional outcome after IVH and/ or SAH. Well-established qualitative scales, such as the modified Fisher scale (mFisher) and the original Graeb scale (oGraeb), are simple to perform and easy to apply in daily practice but show limited predictive power for SDHC. Volumetric quantitative measurement of the total amount of blood for SAH or the intraventricular amount of blood for IVH is considered the gold standard to predict SDHC development but might be impractical in the clinical setting.

Somewhere in between these two options is the modified Graeb scale (mGraeb), which keeps the simplicity of a qualitative scale but adds measures of initial ventricular dilatation, a very interesting feature that may be useful in predicting SDHC. The mGraeb scale was introduced as a predictive tool for SDHC in patients suffering spontaneous IVH. ${ }^{26}$ More recently, it has also been applied for the prediction of delayed cerebral ischemia and functional outcome in patients suffering $\mathrm{SAH}$, although its role for the prediction of SDHC remains unknown.,

In the present study, we investigated the value of the mGraeb scale for the prediction of SDHC after aneurysmal SAH (aSAH). In addition, we compared the predictive capacity of the mGraeb scale with specific previously validated measures, such as volumetry and the mFisher or the oGraeb scale. We hypothesized that mGraeb would show a higher ability to predict SDHC than the classic qualitative scales and would prove at least equally valuable as volumetry in predicting SDHC. Finally, we aimed to define more accurate mGraeb scale cutoff points for the prediction of SDHC.

Finding an accurate and easy tool for SDHC prediction may provide information that may enhance discussions on functional prognosis with patients and their families and might facilitate research on new experimental therapies by defining the most appropriate candidates.

\section{Methods}

The methodology of this report follows the recommendations of the Strengthening the Reporting of Observational Studies in Epidemiology (STROBE) guidelines. ${ }^{38}$

\section{Patients}

A retrospective analysis of all adult patients ( $>18$ years old) admitted to our institution between May 2013 and April 2016 with the diagnosis of aSAH was conducted. Exclusion criteria for this study were nonaneurysmal $\mathrm{SAH}$ $(n=43)$, death within the first 2 weeks $(n=31)$ from hospital admission, hospital admission later than 72 hours from the onset of symptoms $(n=6)$, and surgical evacuation for intraparenchymal hematomas $(n=10)$. Clinical records of patients qualifying for the inclusion criteria were reviewed and the appropriate variables were recorded. The study protocol was approved by the local Clinical Research Ethics Committee from the Hospital Clinic of Barcelona under the requirements of Spanish legislation in the field of biomedical research, the protection of personal data (15/1999), and the standards of Good Clinical Practice, as well as complying with the Helsinki Declaration (1975 and 1983 revisions). Patient consent was not required due to the retrospective nature of the study design and the lack of patient interaction.

\section{Management}

SAH was diagnosed by the presence of a typical clinical history and a positive CT scan showing subarachnoid or intraventricular hemorrhage. In cases in which the CT scan was negative, diagnosis was established after confirming the presence of xanthochromic CSF obtained by lumbar puncture. After the diagnosis was established, emergent angio-CT $(<3$ hours after admission) and urgent angiography $(<24$ hours after admission) were performed to identify the origin of the bleeding. Patients were treated according to national and international guidelines. .,22 $^{4}$

An external ventricular drain (EVD) was placed in patients with a Glasgow Coma Scale score $\leq 13$ and increased ventricular size. Weaning of the EVD was started once the patient was neurologically stable and the CSF was cleared of blood remnants. The opening pressure of the drain was progressively increased until closure. If no neurological changes were observed, a CT scan was routinely performed 24 hours after the closure. If clinical deterioration was observed, an earlier urgent $\mathrm{CT}$ was acquired. In case of any neurological deterioration during the weaning and/or ventricular enlargement on the control 
$\mathrm{CT}$, a definitive shunt was placed. The preferred surgical option, if possible, was a ventriculoperitoneal shunt.

\section{Variables}

Intraventricular and cisternal hemorrhages were evaluated in the initial CT scan. DICOM anonymized images were transferred to a personal computer and processed using the open-source software OsiriX. ${ }^{32}$ The mFisher scale,,$^{11}$ the oGraeb scale,,$^{13}$ the mGraeb scale, ${ }^{26}$ and quantitative blood volumetry were assessed for accuracy and SDHC predictive capability. This procedure was independently performed by two of the authors (S.G. and J.A.H.), who were blinded to demographic data, clinical baseline, and final outcome. Both reviewers repeated each of their assessments 3 months after the first evaluation. The average of the reviewers' scores was obtained for the purpose of analysis.

The mFisher score was evaluated according to the criteria established by Frontera et al. ${ }^{11}$ The oGraeb scale was evaluated according to previous descriptions through the evaluation of the presence of blood in the third, fourth, and right and left lateral ventricles with a maximum possible score of 12 . The third and fourth ventricles receive a maximum score of 2 if they are filled with blood occupying more than $50 \%$ of the ventricle volume. A maximum score of 4 is given for each lateral ventricle if the blood clot occupies more than three-quarters of its volume. ${ }^{26}$ The mGraeb scores are also based on the third, fourth, and right and left ventricles (maximum score 4 for each), together with each of the occipital and temporal horns (maximum score 2 for each). One extra point is added to the score for each of these 8 compartments expanded beyond normal anatomical size. Thus, the maximum possible score is $32 .{ }^{26}$

Quantitative intraventricular and cisternal hemorrhage volumes were calculated using the 3D tool from OsiriX. This tool allows us to create a volume from various consecutive areas, namely regions of interest (ROIs). On the axial CT images, blood clots were manually outlined using the pencil tool in every 5-mm slice. The 3D tool was then activated to obtain the rendering of the blood volume. The volumes within each ventricle and cistern were separately calculated. In the end, intraventricular volume (IV), cisternal volume (CV), and total volume (TV) were registered for analysis purposes.

In addition to blood volume assessment, other variables previously identified as risk factors for SDHC development were also considered. Thus, age, sex, comorbidities (hypertension and diabetes), aneurysm location (anterior or posterior circulation), treatment modality (surgery or endovascular), World Federation of Neurosurgical Societies (WFNS) scale on admission, ${ }^{37}$ and the need for EVD were registered and included for analysis. Hypertension was defined as blood pressure $\geq 140 / 90 \mathrm{~mm} \mathrm{Hg}$ and/or patient on antihypertensive drugs. Diabetes was registered if patients were under antidiabetic treatment and/or had serum glucose $\geq 126 \mathrm{mg} / \mathrm{dl}$ when fasting.

\section{Outcome}

The primary outcome variable was the development of
SDHC, defined by the need for shunt placement according to the management strategy explained above. The overall clinical outcome was evaluated by using the modified Rankin Scale (mRS) 3 months after discharge. Results were then dichotomized into good versus poor outcomes. Good outcome was considered for $\mathrm{mRS}$ scores from 0 to 2 and poor outcome for scores from 3 to 6 .

\section{Statistical Analysis}

Continuous variables were reported as mean (standard deviation) or median (interquartile range) and were compared with the Student t-test, ANOVA, Mann-Whitney, or Kruskal-Wallis tests as appropriate. Categorical variables were compared with the chi-square and Fisher exact tests. Univariate analysis was used to evaluate the clinical and radiological variables associated with SDHC. The selected radiological measures (mGraeb, IV, and TV) were included separately in binary logistic regression models to evaluate their association with the risk of SDHC. In each model, the coefficient of determination $\left(\mathrm{R}^{2}\right.$ and $\left.\mathrm{R}^{2}{ }_{\text {Nagelkerke }}\right)$ and information criteria indicators, such as the Akaike information criterion (AIC) ${ }^{31}$ and the Bayesian information criterion (BIC), were calculated to compare their predictive effects on estimations of the risk of SDHC. A better model is evidenced by a lower AIC, a lower BIC, a higher $\mathrm{R}^{2}$, and a higher $\mathrm{R}^{2}{ }_{\text {Nagelkerke }}$. In addition, a receiver operating characteristic (ROC) curve analysis was performed to determine the optimal cutoff values for each radiological measure for the prediction of SDHC. The maximum value of the Youden Index was selected as such an optimum cutoff point in the ROC curves. Multivariate logistic regression models were used to adjust the estimations of the dichotomized radiological variables based on the best cutoff point for the effect of confounders ( $p$ values lower than 0.25 on univariate analyses) ${ }^{10}$ by using stepwise backward selection with a $\mathrm{p}$ value for variable removal set at 0.10 . The intraobserver and interobserver agreement for each of the techniques was evaluated by analyzing the interclass correlation coefficient and Bland-Altman plots. All statistical analyses were performed using the Stata/IC version 13.1 software for Mac.

\section{Results}

Overall, 168 patients suffering SAH were admitted to our institution between May 2013 and April 2016, of whom a total of 78 patients were finally included in the analysis after applying the aforementioned predefined exclusion criteria, as shown in the flowchart of the study (Fig. 1).

The mean ${ }^{37}$ age of the study patients was 56 (16) years. The majority of the patients were women $(65.4 \%)$, with aneurysms located in the anterior circulation (97.4\%) and treated through endovascular techniques (93.6\%). Hypertension and diabetes were present in $32 \%$ and $3.9 \%$ of the patients, respectively. Most patients were admitted in good clinical condition (WFNS 1, 55.1\%). Overall, 30 (39\%) of the patients required an EVD due to acute hydrocephalus and 19 (24\%) developed SDHC. At the 3-month follow-up, 15 patients $(19 \%)$ had poor functional condition $(\mathrm{mRS} \geq 3)$ and the rate of mortality (mRS 6) was 5\% (Table 1). 


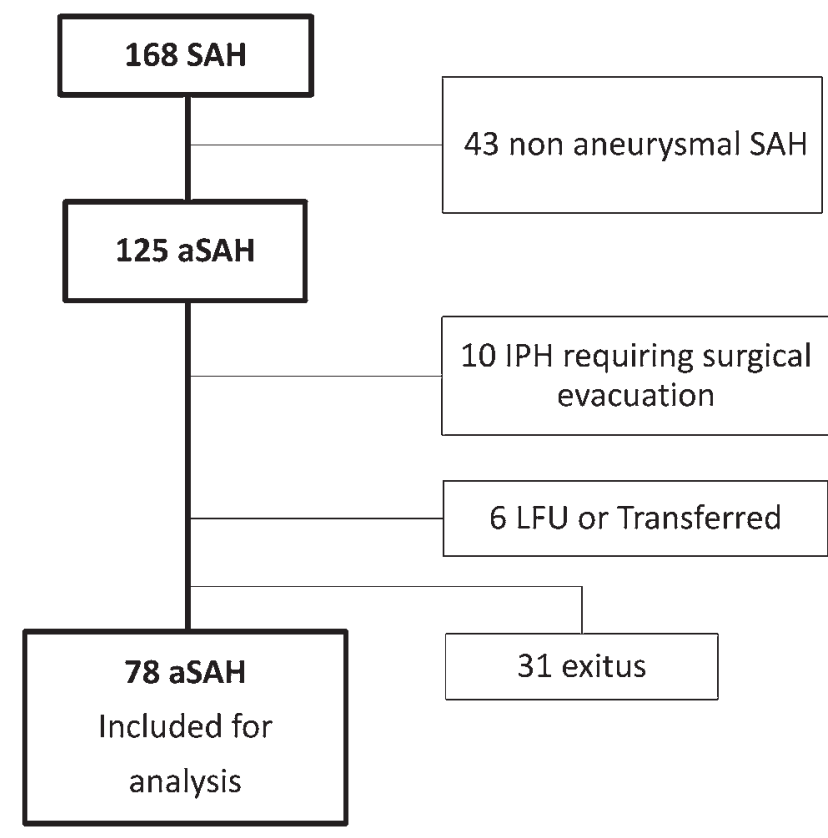

FIG. 1. Flow diagram summarizing patients included in the study. IPH = intraparenchymal hematoma; LFU = lost to follow-up.

The assessment of the early CT scans revealed the following median (IQR) scores in the studied scales: mFisher, 4 (2-4); oGraeb, 1 (0-3); mGraeb, 5.5 (0-11). Regarding blood volumes on the initial CT, the mean ${ }^{37} \mathrm{IV}, \mathrm{CV}$, and TV were $2.5(7.8) \mathrm{ml}, 6.0$ (7.0) $\mathrm{ml}$, and 8.5 (11.8) $\mathrm{ml}$, respectively (Table 1 ).

Total blood volumes in patients requiring a definitive shunt were 3-fold greater than those in patients not suffering SDHC (16.8 ml vs $5.8 \mathrm{ml}$; $\mathrm{p}=0.001)$. Intraventricular blood volume in patients who required a definitive shunt was 8-fold greater than the volume in patients not suffering SDHC (7.29 ml vs $0.91 \mathrm{ml} ; \mathrm{p}=0.0015)$.

The quantitative and qualitative scales were strong predictors of shunt requirement in the univariate analysis: total blood volume $(\mathrm{p}<0.001)$, intraventricular blood volume $(\mathrm{p}=0.001)$, mFisher scale $(\mathrm{p}=0.003)$, oGraeb scale ( $\mathrm{p}<0.001)$, and $\mathrm{mGraeb}$ scale $(\mathrm{p}<0.001)$. Other factors, such as the level of consciousness defined by the WFNS scale and age, were related to the need for shunt placement and achieved statistical significance in the univariate analysis $(\mathrm{p}<0.25)$. When we performed multivariate logistic regression analysis for every scale, only the association of age with the IV had a significant prognostic influence. The remaining models just maintained the quantitative or qualitative scales in the multivariate logistic regression analysis.

ROC and area under the curve (AUC) analyses were implemented to calculate sensitivity and specificity of the models previously described (Table 2 ). In our analysis, the mGraeb scale was the most robust predictor of SDHC, with an AUC value of 0.848 (0.763-0.933). The mGraeb scale had a significantly better AUC than the oGraeb scale $\left(\chi^{2}=4.49 ; \mathrm{p}=0.034\right)$ and mFisher scale $\left(\chi^{2}=7.21 ; \mathrm{p}=\right.$ $0.007)$. However, no statistical difference was found between AUCs of the mGraeb and the quantitative volumet- ric measurement models $\left(\chi^{2}=12.76 ; p=0.23\right)$ (Fig. 2). Youden Index values are shown in Table 3.

On the intra- and interrater reliability analysis, volumetry and all the qualitative scales showed excellent agreement on reassessment, with an interclass correlation coefficient over 0.80 in each case. Moreover, no systematic bias was observed on the Bland-Altman plot for quantitative measurements (Fig. 3).

\section{Discussion \\ Predicting SDHC After SAH}

Blood quantity and location after aSAH might determine both CSF circulation blockages and CSF absorption disruptions leading to SDHC, which has a known negative impact on functional and vital prognoses. Different quantitative techniques and qualitative scales have been implemented to quantify the amount of subarachnoid and intraventricular blood and have previously been validated for aSAH by many authors. ${ }^{3,5-7,24}$ Volumetric measurement of blood volume is more precise and informative ${ }^{17}$ and has been classically considered the most accurate tool to evaluate prognostic implications. ${ }^{18,23}$ However, this quantitative method requires specific software, training, and a research setting not widely available in every clinical location. Furthermore, difficulties differentiating blood from close bony structures when defining the ROI may hamper cistern blood volume measurements. Qualitative scales, on the other hand, are easy and straightforward and can be quickly implemented during the daily clinical routine. Nevertheless, these scales are subject to a certain degree of subjectivity and show limited predictive capabilities. Somewhere in between, the mGraeb scale keeps the simplicity of the qualitative methods but seems to add predictive value by considering the initial ventricular size.

Our study is the first to investigate mGraeb performance for SDHC prediction after aSAH. According to our data, the AUC of the mGraeb scale was superior to those of the other two qualitative methods (oGraeb and mFisher) implemented to account for blood volume (Fig. 2A). However, regarding quantitative measurements, there were no significant differences when mGraeb was compared with volumetry (Fig. 2C). Despite the difference not being statistically significant, the fact that a qualitative scale$\mathrm{mGraeb}$ - proved to have at least as good predictive ability as a quantitative method, which is more time consuming and technically demanding, is a noteworthy finding. Actually, as the $\mathrm{R}^{2}{ }_{\text {Nagelkerke }}$ and AIC analyses show, the mGraeb is the simplest model that best correlates with the development of SDHC (Table 2).

It may seem counterintuitive that a qualitative modelmGraeb-is at least as effective in predicting SDHC as a quantitative volumetric method. Quantitative volumetry is perceived as more accurate and subject to less interobserver variability, which might lead to the idea of better predictive capabilities. However, the mGraeb scale considers blood quantity and location together with enlarged ventricles. Therefore, this scale better defines the amount of blood than do the qualitative mFisher and oGraeb scales, and unlike quantitative volumetry, the mGraeb scale does take into account increased ventricle size. As reported 
TABLE 1. Demographic data of patients included in the study

\begin{tabular}{|c|c|c|c|c|}
\hline Variable & Total $(n=78)$ & No SDHC $(n=59)$ & $\operatorname{SDHC}(n=19)$ & p Value \\
\hline Age in yrs, mean (SD) & $56(16)$ & $53(16)$ & $62(13)$ & 0.050 \\
\hline Female sex, $\mathrm{n}(\%)$ & $51(65)$ & $38(64)$ & $13(68)$ & 0.749 \\
\hline Hypertension, n (\%) & $25(32)$ & $17(29)$ & $8(42)$ & 0.280 \\
\hline Diabetes, $\mathrm{n}(\%)$ & $3(4)$ & $3(5)$ & 0 & 0.316 \\
\hline WFNS grade at admission, $\mathrm{n}(\%)$ & & & & 0.019 \\
\hline 1 & $42(55)$ & $36(62)$ & $6(31)$ & \\
\hline 2 & $15(19)$ & $12(21)$ & $3(16)$ & \\
\hline 3 & $4(5)$ & $1(2)$ & $3(16)$ & \\
\hline 4 & $6(8)$ & $4(7)$ & $2(11)$ & \\
\hline 5 & $10(13)$ & $5(8)$ & $5(26)$ & \\
\hline Aneurysm location, $\mathrm{n}(\%)$ & & & & 0.392 \\
\hline Anterior & $76(97)$ & $58(98)$ & $18(95)$ & \\
\hline Posterior & $2(3)$ & $1(2)$ & $1(5)$ & \\
\hline Treatment modality, $\mathrm{n}(\%)$ & & & & 0.501 \\
\hline Surgery & $5(6)$ & $4(7)$ & $1(5)$ & \\
\hline Endovascular & $73(94)$ & $54(93)$ & $18(95)$ & \\
\hline Fisher scale at baseline CT, n (\%) & & & & 0.003 \\
\hline 1 & $10(13)$ & $10(17)$ & 0 & \\
\hline 2 & $12(15)$ & $10(17)$ & $2(10)$ & \\
\hline 3 & $14(18)$ & $14(24)$ & 0 & \\
\hline 4 & $42(54)$ & $25(42)$ & $17(89)$ & \\
\hline oGraeb score, median (IQR) & $1(0-3)$ & $0(0-3)$ & $3(2-4)$ & $<0.001$ \\
\hline mGraeb score, median (IQR) & $5.5(0-11)$ & $3(0-8)$ & $12(8-20)$ & $<0.001$ \\
\hline \multicolumn{5}{|c|}{ Blood vol in ml at baseline CT, mean (SD) } \\
\hline CV & $6.0(7.0)$ & $4.9(6.4)$ & $9.5(7.9)$ & 0.012 \\
\hline IV & $2.5(7.8)$ & $0.9(2.5)$ & $7.3(14.4)$ & 0.001 \\
\hline TV & $8.5(11.8)$ & $5.8(7.4)$ & $16.8(18.0)$ & $<0.001$ \\
\hline EVD, n (\%) & $30(39)$ & $11(19)$ & $19(100)$ & $<0.001$ \\
\hline Clinical outcome at 3 mos, $n(\%)$ & & & & 0.116 \\
\hline mRS 0-2 & $63(81)$ & $50(85)$ & $13(68)$ & \\
\hline mRS 3-6 & $15(19)$ & $9(15)$ & $6(32)$ & \\
\hline
\end{tabular}

by many authors, acute hydrocephalus is an independent factor directly related to SDHC. ${ }^{8,28,39}$ Blood location, specifically in the third and fourth ventricles, plays a critical role in prognosis and SDHC development. 16,33,39 Therefore, $\mathrm{mGraeb}$ is a robust predictive model because it integrates several independent risk factors for SDHC in one scale: blood quantity, blood location, and acute hydrocephalus. Furthermore, mGraeb is an assessment scale that might be easily and quickly performed during patient evaluation, even in the emergency department, as there is no need for computers or radiological image software. Finally, data on interobserver and intraobserver agreement also show that the $\mathrm{mGraeb}$ scale is a highly reliable measurement tool.

\section{Potential Preventive Therapies for SDHC}

A recent report by Nakatsuka et al. suggested the use of cilostazol, an inhibitor of phosphodiesterase, as a preventive treatment for SDHC in SAH patients. ${ }^{28}$ In a retrospective review of 87 patients they found that the incidence of SDHC was significantly lower in the groups receiving ci- lostazol $(8.3 \%-12.8 \%$ vs $33.3 \%$; $p<0.05)$. Microsurgical fenestration of the lamina terminalis has also been pointed

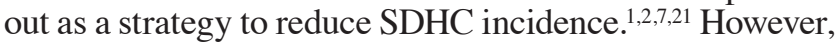
a recent meta-analysis including more than 1900 patients failed to prove a statistically significant association. Nevertheless, the incidence of SDHC in the fenestrated cohort

TABLE 2. Performance of different models for the prediction of SDHC

\begin{tabular}{lcccc}
\hline $\begin{array}{c}\text { Model \& Factors } \\
\text { Included }\end{array}$ & AIC & BIC & AUC $(95 \%$ Cl) & $\begin{array}{c}\mathrm{R}^{2} \text { Nagelkerke } \\
(\%)\end{array}$ \\
\hline mGraeb scale & 66.4 & 71.2 & $0.848(0.763-0.933)$ & 39.7 \\
\hline IV volumetry \& age & 80.5 & 87.5 & $0.757(0.642-0.872)$ & 21.5 \\
\hline TV volumetry & 79.4 & 84.1 & $0.752(0.621-0.882)$ & 20.0 \\
\hline In each model, the AIC, BIC, and coefficient of determination $\mathrm{R}^{2}$ Nagelkerk were \\
calculated to quantify the predictive effects of each parameter on the risk of \\
SDHC. A better model is evidenced by a lower AIC, a lower BIC, and a higher \\
$\mathrm{R}_{\text {Nagelkerke. }}$
\end{tabular}

J Neurosurg Volume 131 • December 2019 

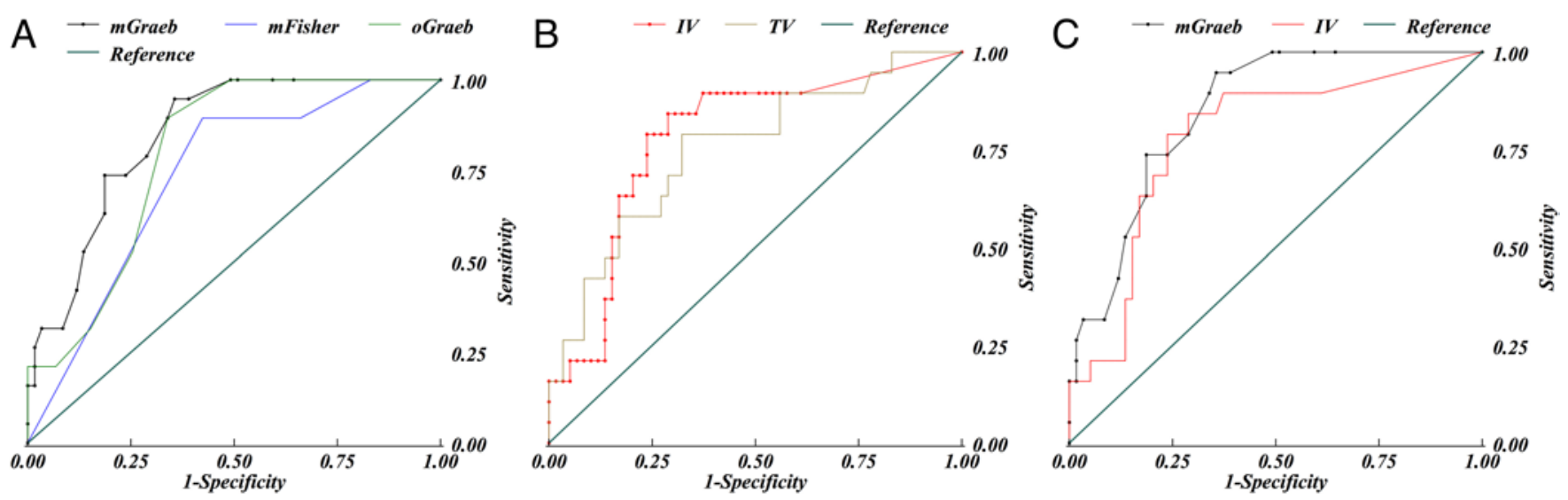

FIG. 2. ROC and AUC analyses for (A) qualitative scales, with mGraeb displaying the greater AUC; (B) volumetric measurements of IV and TV; and (C) volumetric measurements of IV and mGraeb scale.

was lower $(10 \%$ vs $14 \% ; \mathrm{p}=0.089)$ and the majority of the reviewed studies supported its use. ${ }^{21}$ Similarly, CSF clearance by different means has been suggested as an effective technique to prevent SDHC. Endoscopic washout; extensive CSF drainage, whether ventricular or lumbar; and fibrinolysis might contribute to reducing SDHC following intracerebral hemorrhage. ${ }^{19,35}$ However, despite showing trends to better outcomes, these techniques failed to prove a significant effect on mortality, morbidity,${ }^{14}$ and definitive shunt requirement. ${ }^{27}$

Considering all the above, the armamentarium physicians may offer to patients at risk of suffering SDHC is somehow limited. Even if we were able to accurately predict which patients could end up developing chronic hydrocephalus, we still lack proven therapies to reduce the likelihood of eventual shunt dependency. Due to the humble scientific evidence available, we need to be able to detect those patients with a high probability of presenting SDHC. The aforementioned treatment strategies to prevent SDHC should be applied only to those patients in whom there is certainty about their high risk of needing a shunt if no countermeasures are taken.

\section{Cutoff Point of mGraeb and Volumetry for SDHC Predic- tion}

For the two methods showing the best predictive capa-
bilities-mGraeb and volumetry - we have defined a cutoff point that would allow clinical decisions that take into consideration the high specificity needed regarding SDHC. An optimal cutoff point is usually calculated by means of the Youden Index, which is the point in the ROC curve that maximizes sensitivity and specificity. Actually, previous reports have suggested Youden Index values for volumetry and mGraeb used for purposes other than SDHC prediction. ${ }^{9,23}$ Eagles et al. suggested an optimal cutoff point of 3 on the mGraeb scale in order to predict delayed cerebral ischemia. ${ }^{16}$ Lagares et al. found a critical value of $20 \mathrm{ml}$ of total blood volume at which the proportion of patients with poor outcome increased by more than 2 -fold. ${ }^{23}$ Instead of using the cutoff points obtained with the Youden Index, we tried to find a threshold tailored to the very particular circumstances of SDHC and aimed for a more exigent or specific cutoff point. According to our data, patients presenting a score of 12 or more on the initial mGraeb will develop SDHC with more than $85 \%$ specificity. Regarding volumetric measurement, those patients with a TV of 10.3 $\mathrm{ml}$ on the initial CT scan will develop SDHC with more than $80 \%$ specificity.

\section{Limitations}

The present study has some limitations worth mentioning. Patient data were collected retrospectively and some

TABLE 3. Comparison of proposed cutoffs according to the Youden Index versus tailored (or empirical) cutoffs to determine maximum specificity considering the limited scientific evidence for available prophylactic therapies

\begin{tabular}{|c|c|c|c|c|c|c|}
\hline & Cutoff & Specificity (\%) & Sensitivity (\%) & PPV (\%) & NPV $(\%)$ & Accuracy $(\%)$ \\
\hline \multicolumn{7}{|c|}{ Cutoffs of scores $w />80 \%$ specificity \& highest sensitivity } \\
\hline mGraeb scale & $\geq 12$ & 86 & 53 & 56 & 85 & 72 \\
\hline IV volumetry & $\geq 1.3$ & 81 & 63 & 52 & 87 & 77 \\
\hline TV volumetry & $\geq 10.3$ & 83 & 52 & 50 & 84 & 76 \\
\hline \multicolumn{7}{|c|}{ Statistically optimal cutoffs (Youden Index) } \\
\hline mGraeb scale & $\geq 5$ & 64 & 95 & 46 & 97 & 72 \\
\hline IV volumetry & $\geq 0.5 \mathrm{ml}$ & 78 & 68 & 50 & 88 & 76 \\
\hline TV volumetry & $\geq 5.5 \mathrm{ml}$ & 68 & 79 & 43 & 91 & 69 \\
\hline
\end{tabular}

$\mathrm{NPV}=$ negative predictive value; $\mathrm{PPV}=$ positive predictive value. 

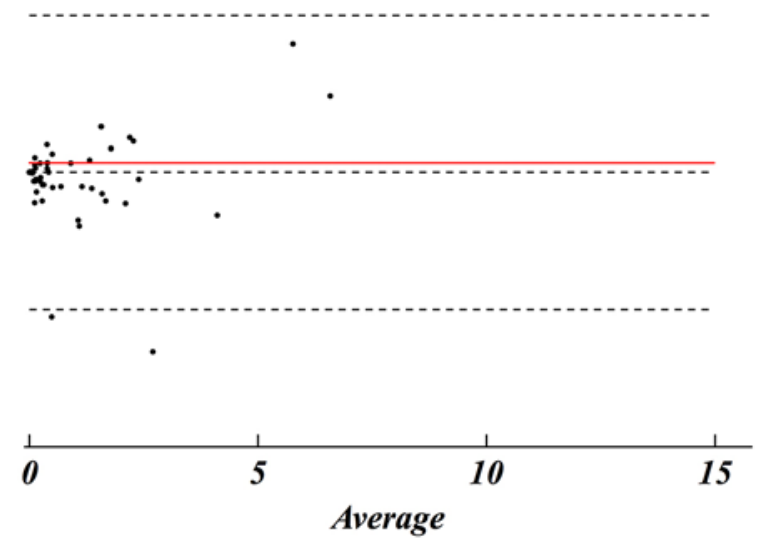

FIG. 3. Bland-Altman plot comparing volumetric measurement agreement between observers. The averages between pairs of measurements are plotted against their differences. The solid red line indicates mean bias (average difference), and the black dashed lines are limits of agreement (95\% confidence limit lines). Figure is available in color online only.

procedures, despite relying on international and national guidelines, may not have been fully standardized. Nevertheless, reviewers implementing the scales based on the initial CT findings were blinded to clinical management and outcome. The fact that more than half of the patients were WFNS grade 1 and $93 \%$ were treated endovascularly may have led to a misrepresentation of some likely risk factors for shunt dependency and might have affected clinical outcomes. Another issue worth considering is the relatively small sample size. However, despite the aforementioned skewness and the number of cases being limited, the cohort was similar to those of many other reported studies on the same topic, and the results reached statistical significance. ${ }^{16,23,25,40}$ In any case, the predictive models proposed here should be validated with a prospective cohort of patients, and thus further studies are still needed.

\section{Conclusions}

This research shows that the mGraeb scale and IV blood volumetry on initial CT of patients suffering aSAH are robust predictors of SDHC development. More interestingly, mGraeb, a relatively simple and easily applicable qualitative scale, showed prediction capabilities equal to those of the more cumbersome quantitative volumetric method. Due to the limited scientific evidence available for treatments aimed at preventing SDHC after aSAH, we recommend mGraeb scores higher than 12 to select patients for those treatments. The proposed mGraeb cutoff points may also serve as a useful prognostic tool since patients with hydrocephalus have worse functional outcome and will likely require an additional intervention for shunt placement.

\section{References}

1. Akyuz M, Tuncer R: The effects of fenestration of the interpeduncular cistern membrane arousted to the opening of lamina terminalis in patients with ruptured ACoA aneurysms: a prospective, comparative study. Acta Neurochir (Wien) 148:725-732, 2006

2. Andaluz N, Zuccarello M: Fenestration of the lamina terminalis as a valuable adjunct in aneurysm surgery. Neurosurgery 55:1050-1059, 2004

3. Bakker AM, Dorhout Mees SM, Algra A, Rinkel GJ: Extent of acute hydrocephalus after aneurysmal subarachnoid hemorrhage as a risk factor for delayed cerebral infarction. Stroke 38:2496-2499, 2007

4. Connolly ES Jr, Rabinstein AA, Carhuapoma JR, Derdeyn CP, Dion J, Higashida RT, et al: Guidelines for the management of aneurysmal subarachnoid hemorrhage: a guideline for healthcare professionals from the American Heart Association/American Stroke Association. Stroke 43:1711-1737, 2012

5. Czorlich P, Mende KC, Vettorazzi E, Regelsberger J, Westphal M, Schmidt NO: Validation of the modified Graeb score in aneurysmal subarachnoid hemorrhage. Acta Neurochir (Wien) 157:1867-1872, 2015

6. Czorlich P, Ricklefs F, Reitz M, Vettorazzi E, Abboud T, Regelsberger J, et al: Impact of intraventricular hemorrhage measured by Graeb and LeRoux score on case fatality risk and chronic hydrocephalus in aneurysmal subarachnoid hemorrhage. Acta Neurochir (Wien) 157:409-415, 2015

7. Dehdashti AR, Rilliet B, Rufenacht DA, de Tribolet N: Shunt-dependent hydrocephalus after rupture of intracranial aneurysms: a prospective study of the influence of treatment modality. J Neurosurg 101:402-407, 2004

8. Dorai Z, Hynan LS, Kopitnik TA, Samson D: Factors related to hydrocephalus after aneurysmal subarachnoid hemorrhage. Neurosurgery 52:763-771, 2003

9. Eagles ME, Jaja BNR, Macdonald RL: Incorporating a modified Graeb score to the modified Fisher scale for improved risk prediction of delayed cerebral ischemia following aneurysmal subarachnoid hemorrhage. Neurosurgery 82:299305, 2018

10. Fagerland MW, Hosmer DW: A goodness-of-fit test for the proportional odds regression model. Stat Med 32:22352249, 2013

11. Frontera JA, Claassen J, Schmidt JM, Wartenberg KE, Temes $\mathrm{R}$, Connolly ES Jr, et al: Prediction of symptomatic vasospasm after subarachnoid hemorrhage: the modified Fisher scale. Neurosurgery 59:21-27, 2006

12. Germanwala AV, Huang J, Tamargo RJ: Hydrocephalus after aneurysmal subarachnoid hemorrhage. Neurosurg Clin $\mathbf{N}$ Am 21:263-270, 2010

13. Graeb DA, Robertson WD, Lapointe JS, Nugent RA, Harrison PB: Computed tomographic diagnosis of intraventricular hemorrhage. Etiology and prognosis. Radiology 143:91-96, 1982

14. Hanley DF, Lane K, McBee N, Ziai W, Tuhrim S, Lees KR, et al: Thrombolytic removal of intraventricular haemorrhage in treatment of severe stroke: results of the randomised, multicentre, multiregion, placebo-controlled CLEAR III trial. Lancet 389:603-611, 2017

15. Heros RC: Acute hydrocephalus after subarachnoid hemorrhage. Stroke 20:715-717, 1989

16. Hughes JD, Puffer R, Rabinstein AA: Risk factors for hydrocephalus requiring external ventricular drainage in patients with intraventricular hemorrhage. J Neurosurg 123:14391446,2015

17. Ibrahim GM, Weidauer S, Macdonald RL: Interobserver variability in the interpretation of computed tomography following aneurysmal subarachnoid hemorrhage. J Neurosurg 115:1191-1196, 2011 
18. Jiménez-Roldán L, Alén JF, Gómez PA, Lobato RD, Ramos A, Munarriz PM, et al: Volumetric analysis of subarachnoid hemorrhage: assessment of the reliability of two computerized methods and their comparison with other radiographic scales. J Neurosurg 118:84-93, 2013

19. Johnson JR, Idris Z, Abdullah JM, Alias A, Haspani MS: Prevalence of shunt dependency and clinical outcome in patients with massive intraventricular haemorrhage treated with endoscopic washout and external ventricular drainage. Malays J Med Sci 24:40-46, 2017

20. Ko SB, Choi HA, Carpenter AM, Helbok R, Schmidt JM, Badjatia N, et al: Quantitative analysis of hemorrhage volume for predicting delayed cerebral ischemia after subarachnoid hemorrhage. Stroke 42:669-674, 2011

21. Komotar RJ, Olivi A, Rigamonti D, Tamargo RJ: Microsurgical fenestration of the lamina terminalis reduces the incidence of shunt-dependent hydrocephalus after aneurysmal subarachnoid hemorrhage. Neurosurgery 51:1403-1413, 2002

22. Lagares A, Gómez PA, Alén JF, Arikan F, Sarabia R, Horcajadas A, et al: [Aneurysmal subarachnoid hemorrhage: group of study of cerebrovascular pathology of the Spanish Society of Neurosurgery management guideline.] Neurocirugia (Astur) 22:93-115, 2011 (Span)

23. Lagares A, Jiménez-Roldán L, Gomez PA, Munarriz PM, Castaño-León AM, Cepeda S, et al: Prognostic value of the amount of bleeding after aneurysmal subarachnoid hemorrhage: a quantitative volumetric study. Neurosurgery 77:898-907, 2015

24. Mayfrank L, Hütter BO, Kohorst Y, Kreitschmann-Andermahr I, Rohde V, Thron A, et al: Influence of intraventricular hemorrhage on outcome after rupture of intracranial aneurysm. Neurosurg Rev 24:185-191, 2001

25. Mehta V, Holness RO, Connolly K, Walling S, Hall R: Acute hydrocephalus following aneurysmal subarachnoid hemorrhage. Can J Neurol Sci 23:40-45, 1996

26. Morgan TC, Dawson J, Spengler D, Lees KR, Aldrich C, Mishra NK, et al: The modified Graeb score: an enhanced tool for intraventricular hemorrhage measurement and prediction of functional outcome. Stroke 44:635-641, 2013

27. Murthy SB, Awad I, Harnof S, Aldrich F, Harrigan M, Jallo J, et al: Permanent CSF shunting after intraventricular hemorrhage in the CLEAR III trial. Neurology 89:355-362, 2017

28. Nakatsuka Y, Kawakita F, Yasuda R, Umeda Y, Toma N, Sakaida H, et al: Preventive effects of cilostazol against the development of shunt-dependent hydrocephalus after subarachnoid hemorrhage. J Neurosurg 127:319-326, 2017

29. Nam KH, Hamm IS, Kang DH, Park J, Kim YS: Risk of shunt dependent hydrocephalus after treatment of ruptured intracranial aneurysms: surgical clipping versus endovascular coiling according to Fisher grading system. J Korean Neurosurg Soc 48:313-318, 2010

30. O'Kelly CJ, Kulkarni AV, Austin PC, Urbach D, Wallace MC: Shunt-dependent hydrocephalus after aneurysmal subarachnoid hemorrhage: incidence, predictors, and revision rates. Clinical article. J Neurosurg 111:1029-1035, 2009

31. Quiñones-Hinojosa A, Chang EF, Chaichana KL, McDermott MW: Surgical considerations in the management of falcotentorial meningiomas: advantages of the bilateral occipital transtentorial/transfalcine craniotomy for large tumors. Neurosurgery 64 (5 Suppl 2):260-268, 2009
32. Ratib O, Rosset A, Heuberger J: Open source software and social networks: disruptive alternatives for medical imaging. Eur J Radiol 78:259-265, 2011

33. Rincon F, Gordon E, Starke RM, Buitrago MM, Fernandez A, Schmidt JM, et al: Predictors of long-term shunt-dependent hydrocephalus after aneurysmal subarachnoid hemorrhage. Clinical article. J Neurosurg 113:774-780, 2010

34. Sheehan JP, Polin RS, Sheehan JM, Baskaya MK, Kassell NF: Factors associated with hydrocephalus after aneurysmal subarachnoid hemorrhage. Neurosurgery 45:1120-1128, 1999

35. Staykov D, Kuramatsu JB, Bardutzky J, Volbers B, Gerner ST, Kloska SP, et al: Efficacy and safety of combined intraventricular fibrinolysis with lumbar drainage for prevention of permanent shunt dependency after intracerebral hemorrhage with severe ventricular involvement: a randomized trial and individual patient data meta-analysis. Ann Neurol 81:93-103, 2017

36. Stienen MN, Weisshaupt R, Fandino J, Hildebrandt G, Studerus-Germann A, Schatlo B: Characteristics of patients without neuropsychological deficits following aneurysmal subarachnoid haemorrhage. Acta Neurochir Suppl 120:125129,2015

37. Teasdale GM, Drake CG, Hunt W, Kassell N, Sano K, Pertuiset B, et al: A universal subarachnoid hemorrhage scale: report of a committee of the World Federation of Neurosurgical Societies. J Neurol Neurosurg Psychiatry 51:1457, 1988

38. Vandenbroucke JP, von Elm E, Altman DG, Gøtzsche PC, Mulrow CD, Pocock SJ, et al: Strengthening the Reporting of Observational Studies in Epidemiology (STROBE): explanation and elaboration. Epidemiology 18:805-835, 2007

39. Wilson CD, Safavi-Abbasi S, Sun H, Kalani MY, Zhao YD, Levitt MR, et al: Meta-analysis and systematic review of risk factors for shunt dependency after aneurysmal subarachnoid hemorrhage. J Neurosurg 126:586-595, 2017

40. Yu H, Zhan R, Wen L, Shen J, Fan Z: The relationship between risk factors and prognostic factors in patients with shunt-dependent hydrocephalus after aneurysmal subarachnoid hemorrhage. J Craniofac Surg 25:902-906, 2014

\section{Disclosures}

The authors report no conflict of interest concerning the materials or methods used in this study or the findings specified in this paper.

\section{Author Contributions}

Conception and design: Torné. Acquisition of data: García, Hoyos. Analysis and interpretation of data: Torné, Amaro. Drafting the article: Torné, García, Rodríguez-Hernández. Critically revising the article: all authors. Reviewed submitted version of manuscript: all authors. Approved the final version of the manuscript on behalf of all authors: Torné. Statistical analysis: Torné. Administrative/technical/material support: Rodríguez-Hernández, López, Enseñat. Study supervision: Torné, Enseñat.

\section{Correspondence}

Ramon Torné: Hospital Clinic de Barcelona, Barcelona, Spain. ramtorne@hotmail.com. 